\title{
Behavior patterns in hormonal treatments using fuzzy logic models
}

\author{
J. G. Enríquez ${ }^{1}$ - V.Cid ${ }^{1}$ - N. Muntaner ${ }^{1}$ - J. Aroba $^{2}$ - J. Navarro ${ }^{3}$. \\ F. J. Domínguez-Mayo ${ }^{1}$ - M. J. Escalona ${ }^{1}$ - I. Ramos ${ }^{1}$
}

\begin{abstract}
Assisted reproductive technologies are a combination of medical strategies designed to treat infertility patients. Ideal stimulation treatment has to be individualized, but one of the main challenges which clinicians face in the everyday clinic is how to select the best medical protocol for a patient. This work aims to look for behavior patterns in this kind of treatments, using fuzzy logic models with the objective of helping gynecologists and embryologists to make decisions that could improve the process of in vitro fertilization. For this purpose, a real-world dataset composed of
\end{abstract}

one hundred and twenty-three (123) patients and five hundred and fifty-nine (559) treatments applied in relation to such patients provided by an assisted reproduction clinic, has been used to obtain the fuzzy models. As conclusion, this work corroborates some known clinic experiences, provides some new ones and proposes a set of questions to be solved in future experiments.

Keywords Hormonal treatments · IVF · Fuzzy logic · Data mining

\section{Introduction}

Infertility of couples is nowadays considered an important medical and social problem which is subject of special interest by medical doctors and biologists. Intensive research is being done in this field in order to improve both the techniques and treatments applied to improve the results and welfare of patients (Morales et al. 2008). According to the World Health Organization (WHO), infertility affects more than 80 million people worldwide, moreover, according to data presented at Spanish Society of Fertility (Sociedad Española de Fertilidad, SEF) (SEF 2016), the infertility rate in Spain represents between 15 and 17\% of the population. ART, which include IVF, is a set of medical strategies that enables infertile couples to achieve successful pregnancy.

Figure 1 describes the activity diagram that represents the seven phases of which IVF procedure consists of: patient personalized assessment, ovarian stimulation, oocyte retrieval, oocyte insemination, laboratory embryo culture, embryo transfer and pregnancy test. At each step of the procedure, many dependent and independent variables may impact the outcome, i.e., pregnancy (Jurisica et al. 1998; Cotton et al. 2005). 


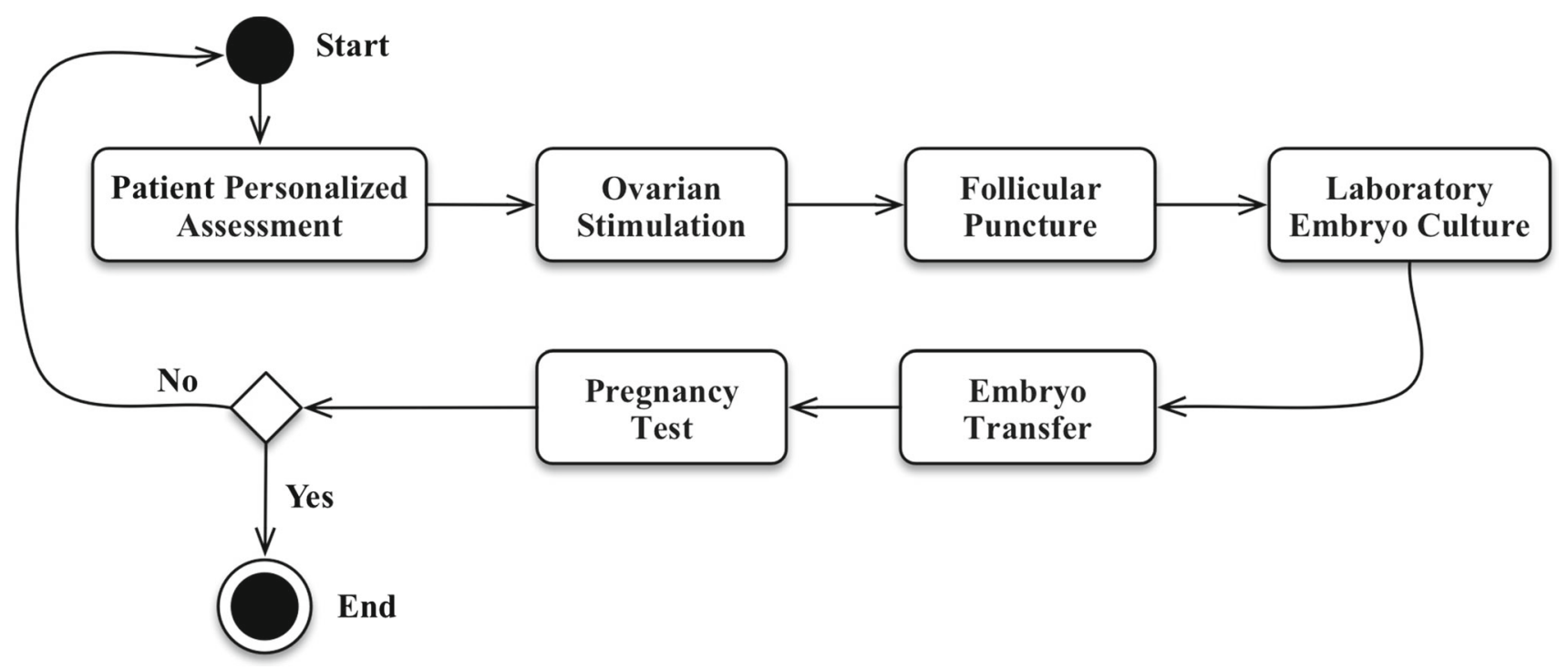

Fig. 1 IVF procedure

In patient personalized assessment phase, a personal assessment of each patient is performed to determine all the important data for applying the IVF procedure such as: their age, ovarian reserve, body mass index or personal and gynecological history, and a set blood test and clinical exams. All these series of medical studies let the doctor choose the most appropriate treatment for each patient. The aim of ovarian stimulation phase is the ovarian stimulation with hormones that induce the formation of a greater number of follicles, within which there is an oocyte. This procedure is necessary to increase the chances of success because as naturally, women just make one follicle and therefore one oocyte each menstrual cycle. The duration of treatment is about 1020 days, depending on the used protocol and the speed of response of each patient. During this process, serial ultrasounds and blood tests are required in which the amount of estradiol is checked to determine if the growth and evolution of the follicles is appropriate.

When evidenced by ultrasound show up that follicles have reached the appropriate size and it is considered that there are an adequate number of oocytes already available, the oocyte retrieval is programmed $36 \mathrm{~h}$ after the administration of subcutaneous hCG (human chorionic gonadotropin) which induce the final maturation of the oocytes. Once oocytes and sperm are collected, and the procedure of oocyte insemination is performed. The resulting embryos are observed in the laboratory every day for 3-6 days, and they are classified according to their morphology in the embryo culture laboratory phase. Those who become blocked in their development will be discarded because they will be considered non-viable.

When one to three embryos are ready, it is performed the embryo transfer phase. These phase consists in an operation performed to the patient in which the best quality embryos is introduced in the uterine cavity with the help of a cannula specially designed for it. A blood pregnancy test is carried out between 11 and 15 days after the embryo transfer to confirm if pregnancy has been achieved.

Ovarian stimulation and IVF are complex and expensive medical strategies, and they involve a disturbing psychological impact for patients. In Lawson et al. (2014) authors performed a comparison between patients with cancer and infertile couples and they conclude that levels of anxiety and depression are higher in cancer patients before the treatment; however, infertile patients' anxiety and depressive symptoms increase more than the cancer ones across treatment and this increase was not related to time between registration for IVF and oocyte retrieval or the medical aspects of treatment.

This work is based on a real-world dataset (with 123 patients and 559 treatments applied in relation to such patients) to which data mining techniques has been applied. Data mining has a huge potential to help medical specialties to get meaningful information from their databases. Tools based on this technology allow predicting future tendencies and behaviors focused on this stored knowledge; they explore the databases looking for hidden patterns, extracting predictable information that cannot be found by means of classical statistical techniques. Concretely, we have applied the developed tool Predictive Fuzzy Rules Generator (PreFurGe) (Aroba 2003). It is a data mining tool dealing with fuzzy clustering, whose methodology is based on Sugeno and Yasukawa (1993) proposal which has been improved to be able to apply it to multi-parametric quantitative databases with more than a unique output variable.

In this paper, through the use of fuzzy logic applied to the real-world dataset exposed before, we try to look for behavior 
patterns that will help gynecologist and embryologist to make decisions when IVF treatments are applied for a concrete type of patient.

The remainder of this paper is organized as follow: Sect. 2 describes the related work that have been done in this research line, in Sect. 3, the data used, the fuzzy rules generation methodology are described and a set of experiments are set up. Section 4 describes and analyzes the experiments proposed in Sect. 2. The paper ends with conclusions and future works.

\section{Related work}

Data mining (extracting hidden but predictable information from large databases) has a huge potential to recover meaningful information from databases. Tools based on this technology enable the prediction of future tendencies and behaviors, based on the stored data from a system, and go further than the knowledge provided by classical statistical techniques (Hand 1998; Glymour et al. 1996; Nakajima 1996).

From a practical point of view, it is interesting to be able to analyze experimental data qualitatively, that is, by considering natural language, using rules relating to the main magnitudes of a system. By means of this procedure, data description can be interpreted in the same way as a human expert would do, thanks to the use of fuzzy logic (Furuhashi and Suzuki 2001; Guillaume 2001).

Fuzzy logic Zadeh (1965) operates using rules of reasoning which are very close to the imprecise, intuitive, human way of thinking. Fuzzy logic allows a variable to be characterized without specifying a precise value, something which is not possible with classical logic. Traditionally, in classical logic, membership of one class or set is binary, i.e., an element is either a member or not of a set. By contrast, fuzzy logic, by means of a membership function, allows an association of each sample with a set, according to a certain grade of membership.

Also, fuzzy logic provides a systematic calculation for processing knowledge that is uncertain, imprecise or with incomplete information (Bui et al. 2012). Nowadays, there are some fuzzy logic models studies which provide different solutions in many medical areas: Lopes et al. (2015) describe a decision support system modeled on fuzzy logic that defines lower urinary tract dysfunctions (LUTD) using the terminology of the International Continence Society. This methodological study aimed to develop a model that uses the maxmin composition of fuzzy relations that can perform differential diagnoses of LUTD. The model was capable of determining a diagnosis in full or partial agreement with the medical report. This proposed model is very simple, and it shows interesting results. Many different works have applied fuzzy logic to different clinical environments. Rane (2015) proposed a clinical decision support system. This system statistically models the patients illnesses and symptoms before recommending treatments. The proposed model is designed to use data mining techniques such as: neural networks, decision trees and analysis of clustering patterns among others, in order to try to improve the survival of human life. In the system proposed by Josefiok and Sauer (2015), authors improved practitioners performance in certain areas in the field of neurology because of the differential diagnoses complexity using fuzzy logic. Panavaranan and Wongsawat (2013) described the pain phenomenon. An acute pain is one of the most severe pain for patient, and it also has difficulty treatment for therapist. The authors proposed a use of intelligent system based on a fuzzy logic algorithm and kernel support vector machine in order to estimate pain level and classify a state of pain.

Yilmaz and Ayan (2013) expound that stress triggers cancer types as well as the possibility of developing cancer may be decreased and people could take measures against it with fuzzy logic models. The authors concluded that thanks to this type of study, people will have the chance to take measures against developing cancer and the rate of suffering from cancer may be decreased. Yilmaz and Ayan (2011) described that the use of fuzzy logic model to discover the risks of getting cancer for selected pilot people could improve to improve tackle this situation. The authors presented a model that makes a prediagnosis for people who possibly can have risk of getting cancer due to working conditions or living standards.

Other totally different fields of clinical environments such as: traumatology, dietetics, stress treatments or cardiology have also been explored. Shamim et al. (2009) described the significant number of patients who fail to benefit from surgery for symptomatic lumbar disk herniation. They used a fuzzy logic system for identifying patients unlikely to improve after disk surgery and proposed it as a prediction tool of this cases. Miyahira and Araujo (2008) presented a fuzzy obesity index for being used as an alternative in obesity treatment and bariatric surgery indication. The authors presented a fuzzy mechanism for evaluating obesity that yields a fuzzy obesity index for its evaluation and treatment. Also this mechanism allows to build up decision support system. Tartarisco et al. (2012) described an integrated processing approach based on an autoregressive model, artificial neural networks and fuzzy logic modeling that allows stress conditions to be automatically identified with a mobile setting analyzing features of the electrocardiographic signals and human motion. Borges and Brusamarello (2015) described a method to avoid false alarms in hospitals caused by electromagnetic interference or motion artifacts, for example, heart rate. In this work, a set of algorithms were proposed to fuse the information from the electrocardiogram, arterial blood pressure and pho- 
toplethysmogram. The proposed algorithms showed good performance and could be useful in bedside monitors.

It is possible to note that lots of different fuzzy logic techniques applied to lots of different clinic environments have been studied. However, it has been very difficult to find recent works related to the topic of this paper: the application of fuzzy logic to the hormonal treatments, a very important medical and social problem, which is increasingly affected. In this sense, it was decided to use a well-known fuzzy logic tool of the authors of this paper: PreFuRGe (Aroba 2003). This tool has allowed the discovery of new, very relevant (and sometimes hitherto unknown) information, using datasets drawn from various diverse contexts like for example: software engineering (Aroba et al. 2008) or systems identification in control (Gegúndez et al. 2008). All the results obtained have been validated by experts in their respective fields, confirming the veracity and importance of the qualitative information generated by the tool.

\section{Materials and methods}

The data that have been used for this work comes from the INEBIR (Instituto para el Estudio de la Biologa de la Reproduccin Humana) fertility clinic, at the Victoria Eugenia hospital. This clinical unit is composed of seven subunits: major ambulatory surgery, gynecology, endometriosis, genetics, infertility, embryo laboratory and psychology. The R\&D unit focuses its attention in four research lines: fertility preservation, biomarkers, cancer and endometriosis. This paper is the result of a new project with the collaboration of the IWT2 (Ingenieria Web y Testing Temprano) research group of the University of Seville, based on the analysis of the clinical databases used at INEBIR.

The protocol used in this work has been divided in three phases: data preparation, data capture and execution and analysis of results. In data preparation phase, the data provided by INEBIR were analyzed and the different experiments were set up. In data execution phase, the data mining tool PreFurGe was applied to the experiments to obtain different fuzzy rules and, finally, in analysis phase the results obtained from the previous phase were analyzed.

\subsection{Fuzzy rules generation methodology}

In this section, fuzzy rules generation methodology is presented. It is composed of two main pillars: the fuzzy clustering and the PreFurGe methodology.

\subsubsection{Fuzzy clustering}

A clustering algorithm Kaufman and Rousseeuw (2009) groups a set of data in such a way that objects in the same group (cluster) are more similar to each other than to those in other clusters. Classical clustering algorithms generate a partition of the population in a way that each element is assigned to a cluster. These algorithms use the so-called rigid partition derived from the classical sets theory: the membership grade of each element can only take values 0 or 1 : with zero indicating null membership and one indicating whole membership to each one of the cluster. On the other hand, fuzzy partition Hathaway and Bezdek (1993) holds the same conditions and constraints for its elements, but in this case, the membership grade can take real values between zero and one (partial membership grade). Therefore, elements may belong to more than one group, so that the selecting and clustering capacity increases. A well-known general-purpose fuzzy clustering algorithm is the so-called fuzzy c-means (FCM). (Hathaway and Bezdek 1993; Fukuyama and Sugeno 1989; Hoppner and Klawonn 2003). It is based on the minimization of distances between two data points and the prototypes of cluster centers (c-means).

\subsubsection{PREFURGE methodology}

The first step, before data processing and obtaining the fuzzy rules, consists in establishing the parameters of interest, that is, the variables whose behavior researchers want to study (i.e., pregnancy result o number of cells). These parameters will assume the role of consequents in the future fuzzy rules and, as a result, the rest of the parameters will play the role of antecedents (Fig. 2).

In general terms, PreFuRGe analyses and automatically classifies the data of the experiments so that the consequent parameters are fuzzy clustered. An optimum number of fuzzy clusters are obtained by taking into account the variance of the data in a cluster and that of the clusters themselves, as is described in Fukuyama and Sugeno (1989). Once the consequent parameters have been clustered, each cluster is projected onto the antecedent space, by using the Sugeno and Yasukawas proposal described in Sugeno and Yasukawa (1993). As a result, the membership grade of the antecedents to each fuzzy cluster is determined and from them, the member functions of the antecedents are obtained. Finally, the fuzzy rules are built by considering the membership functions obtained in the fuzzy clustering and fuzzy projection stages, previously described.

Following the application of the fuzzy clustering and the antecedent space projection, the membership function of both antecedents and the consequents will be represented by different types of trapezium (see Fig. 2). Then, PreFuRGe graphically represents the relations between these membership functions yielding the fuzzy rules that qualitatively model the system.

It is important to note that the graphic output provided by PreFuRGe enables an easy interpretation of the fuzzy rules 

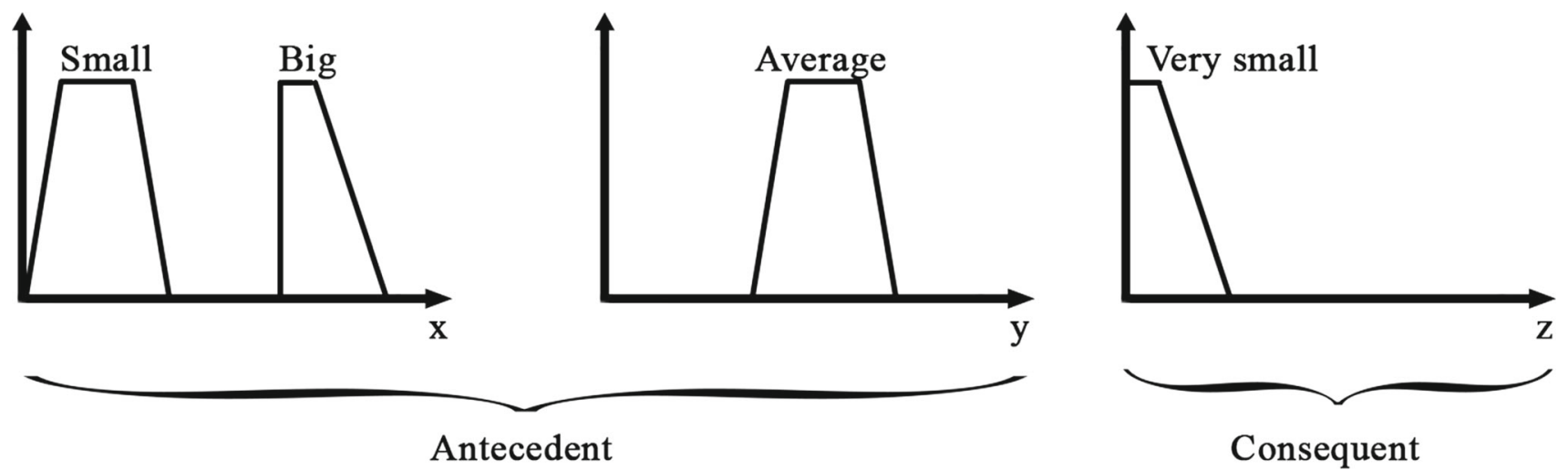

Fig. 2 Example of If-Then fuzzy rule
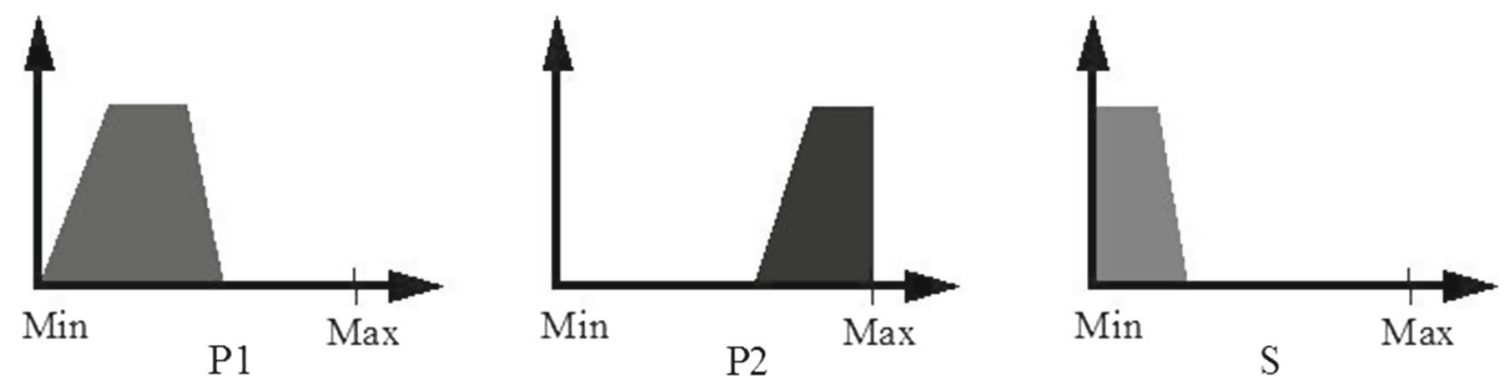

Fig. 3 Example of fuzzy rule
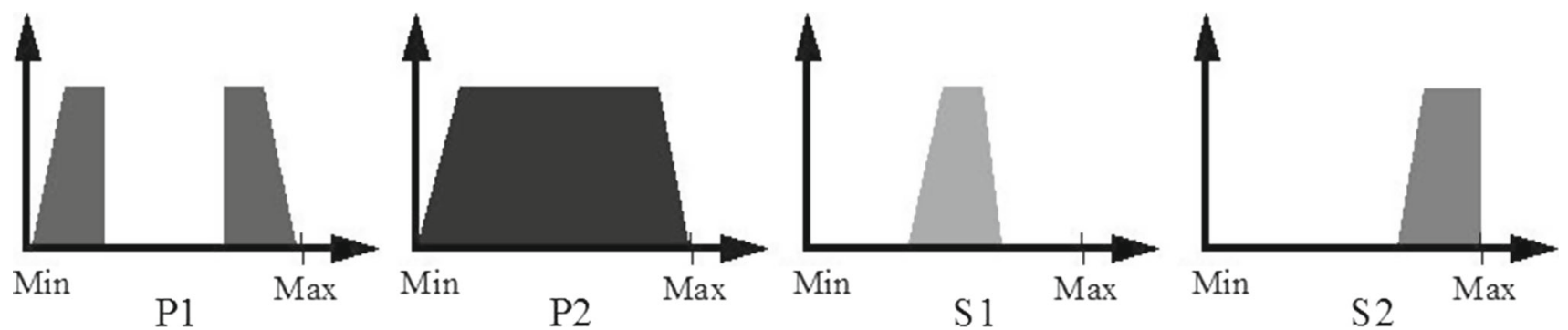

Fig. 4 Example of fuzzy rule with multiple projection

in a natural language. For instance, Figs. 3 and 4 show two examples of fuzzy rules generated by PreFuRGe. In these rules, the fuzzy set assigned to each parameter is represented by a trapezium. The parameter values are represented on the $x$ axis of each fuzzy set, and the value of membership grade to a cluster on the $y$ axis.

In the rule presented in Fig. 3, the parameters of the antecedent are: $\mathrm{P} 1$ and $\mathrm{P} 2$; and the consequent is: S. This fuzzy rule would be interpreted as follows:

IF P1 is bigger than small and lower than average AND $P 2$ is big THEN $S$ is small.

Furthermore, it is remarkable that PreFuRGe can also generate rules where the consequent includes more than one parameter. Moreover, when applying PreFuRGe to a dataset, it is possible to obtain multiple projections (two fuzzy sets related to an input parameter), or even find a projection that cover all possible values of a parameter. For instance, in the fuzzy rule represented in Fig. 4, a consequent of two parameters (S1 and S2) is considered. In this case, the antecedent parameters are: $\mathrm{P} 1$ and $\mathrm{P} 2$. Observe that a multiple projection is represented in $\mathrm{P} 1$, so that this parameter can take two different types of values in this rule. Also note that, $\mathrm{P} 2$ can take values in the whole universe of discourse. Therefore, this parameter does not contribute to the linguistic interpretation of the rule, because it does not present a defined behavior pattern. As a consequence, this fuzzy rule can be interpreted as follows:

IF P1 is small or big THEN S1 is average AND S2 is big.

\subsection{Experiments}

Once understood the environment in which we worked, the clinical performance was divided in: the clinical process by itself, where doctors examine the patients and order a 
set of clinical tests and the laboratory process where the results obtained from the tests are analyzed. For this work, two experiments and a set of configurations for each experiment were carried out, one based on the clinical process and another based on the laboratory process.

The first experiment aims to look for a relation between the result of pregnancy of a treatment depending on different characteristics and hormones of the patient during the treatment. The important data established for the experiment were: the maximum value of estradiol hormone in patients before the follicular puncture, the number of cells in the second day of treatment, the patient's age and finally, the result of pregnancy, whether positive or negative.

For this experiment, four combinations were analyzed with different objectives:

- Combination 1 As antecedent, the number of cells, the patients age and the estradiol value were used and the pregnancy result was set up as consequence.

- Combination 2 As antecedent the number of cells and the estradiol value were used, and the pregnancy result and the patients age were set up as consequence.

- Combination 3 As antecedent, the estradiol value, the patients age and the pregnancy result were used, and the number of cells was set up as consequence.

- Combination 4 As antecedent, the number of cells, the patients age and the pregnancy result as were used, and estradiol value was set up as consequence.

The second experiment aims to look for a behavior pattern that relates the medicines supplied to the patient during treatment and the number of cells of such patient. The important data established for the experiment were: the number of cells analyzed before the follicular puncture and the medicines supplied (first medication, stimulation medication and human chorionic gonadotropin (hCG) medication). In this case, one combination was analyzed:

- Combination 1 As antecedent, the first medication, stimulation medication and hCG values were used and the number of oocytes was set up as consequence.

\subsection{Data preparation}

Database of INEBIR is composed of a large number of tables unrelated between them and a large number of records. Therefore, the first step was conducting a study of the database with the aim of eliminating tables that, a priori, were not required for the study. All tables with their corresponding fields and the records that composed the database were analyzed. Finally, from the one hundred and twenty-six tables that composed the database, it remained twenty-six tables potentially interesting.
Through the study of the database, it was concluded that the important data came from a set of table views that provided information to the INEBIRs system for the management of the treatments lifecycle. Thus, extraction of data necessary to perform the experiments described in the previous section, based on the table views mentioned above and the relationship with the main remaining tables resulted from the first step was performed.

The final dataset ready to be processed by PreFurGe was composed of a set of one hundred and twenty-three (123) patients and five hundred and fifty-nine (559) applied treatments with the relevant data for the experiments described previously.

\section{Results}

Once the data were retrieved and the experiments were set up, the PreFurGe was applied. For each combination of each experiment, the set of rules and some notes have been described. Each graphical row obtained from the application of the tool represents a rule. Each combination has been named as:

"EXPexperimentNumber

COMBcombinationNumber" (i.e., EXP2_COMB1)

- EXP1_COMB1 (Antecedent: Number of Cells - Age - Estradiol, Consequence: Pregnancy Result) (Fig. 5). Note Since the consequent is a binary attribute that only takes the values 0 (positive) and -1 (negative), results will only obtain two fuzzy rules (R1 and R2).

\section{- Rules}

- $\mathbf{R} 1$ shows that the positive outcome of pregnancy (value 0 ) occurs in women with any number of cells, with any concentration of estradiol, and almost any age, except maximum aged.

- R2rule it shows that the negative result of pregnancy $(-1)$ occurs in women with a number of cells ranging from very low to greater than medium (without reaching maximum values), with a concentration similar estradiol number of cells, and more than 27 years old (approximately). That is, the negative result does not occur in young women 20-27 years of (approximately), high number of cells with high concentration of estradiol.

- EXP1_COMB2 (Antecedent: Number of Cells - Estradiol, Consequence: Pregnancy Result + Age) (Fig. 6). Note: The consequence values (Result Pregnancy + Age) have been normalized to range $(0,10)$ to avoid distortions in the process of fuzzy clustering. mize 


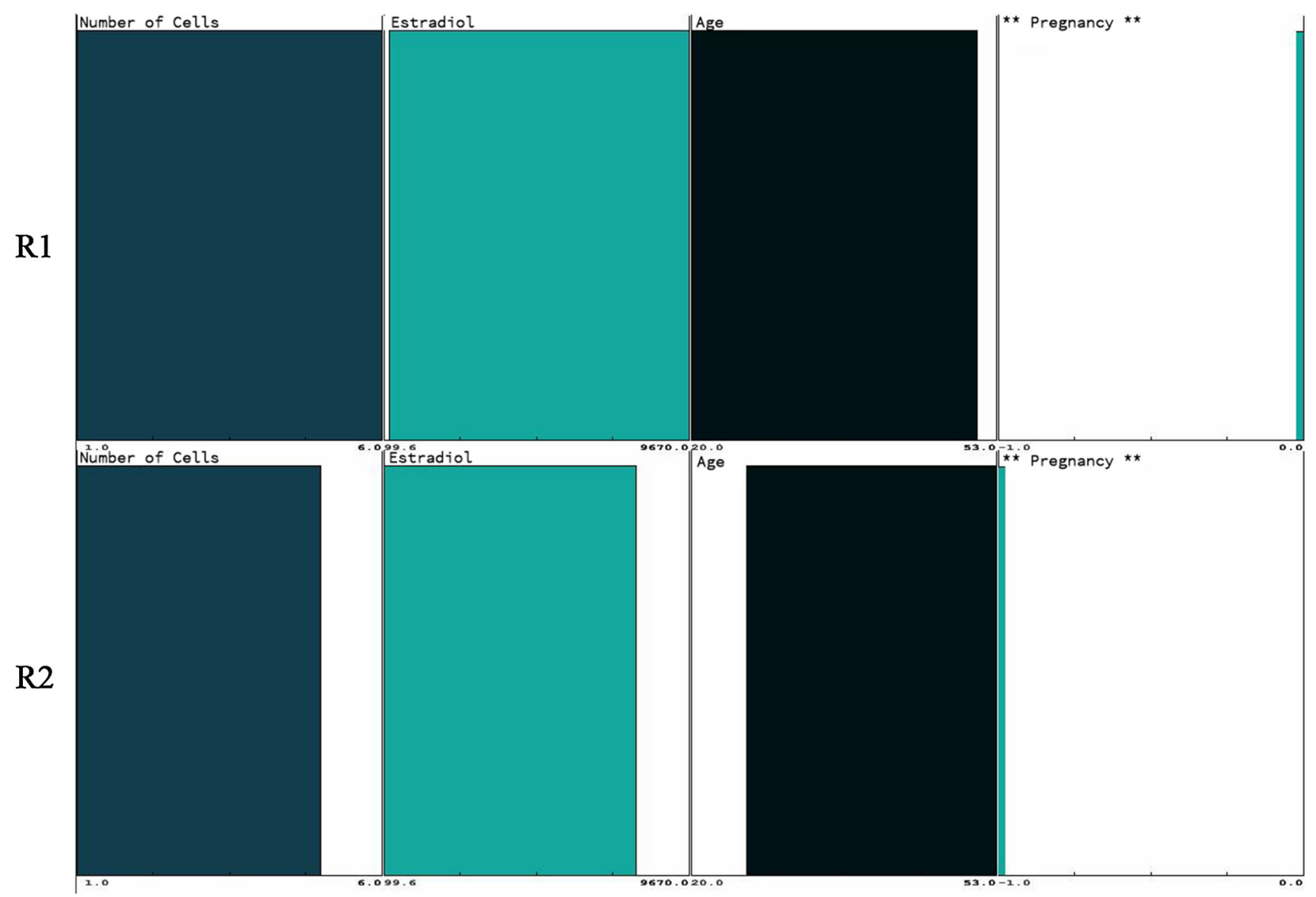

Fig. 5 Rules from experiment 1 -combination 1

\section{- Rules}

- R1 provides an interesting result because it shows a clear pattern of qualitative behavior in women with positive pregnancy outcome (0) and aged ranging from very low to somewhat less than medium (20-30years old approx.), as is corresponding with women with almost any number cell (not reaching the maximum) and with a concentration of estradiol slightly less than medium (but without being very low).

- R2 shows that women with negative pregnancy outcome $(-1)$ and aged over 25 years old (approx.) possess a number of cells and an estradiol concentration ranging from very low values to somewhat lower values than the maximum.

- R3 shows that women with pregnancy outcome positive with medium aged (about 35 years old) have a number of cells ranging from very low to medium and almost any concentration of estradiol (without reaching very low).

- R4 shows that women with positive pregnancy result aged higher than average but not quite high (between 40 and 45 years old) have any number of cells and a concentration of estradiol that takes low to medium values.

- EXP1_COMB3 (Antecedent: Estradiol - Age - Result Pregnancy, Consequence: Number of Cells) (Fig. 7). Note: The consequence values (Result Pregnancy + Age) have been normalized to range $(0,10)$ to avoid distortions in the process of fuzzy clustering.

\section{- Rules}

- R1 does not provide relevant information due to low values of number of cells is given in women with any value in all the attributes of the antecedent.

- $\mathbf{R 2}$ indicates that high values of number of cells is given in women with an estradiol concentration from very low to medium, aged greater than medium-low and lower than very high and with any pregnancy outcome.

- EXP1_COMB4 (Antecedent: Number of Cells - AgePregnancy Result, Consequence: Estradiol) (Fig. 8).

\section{- Rules}




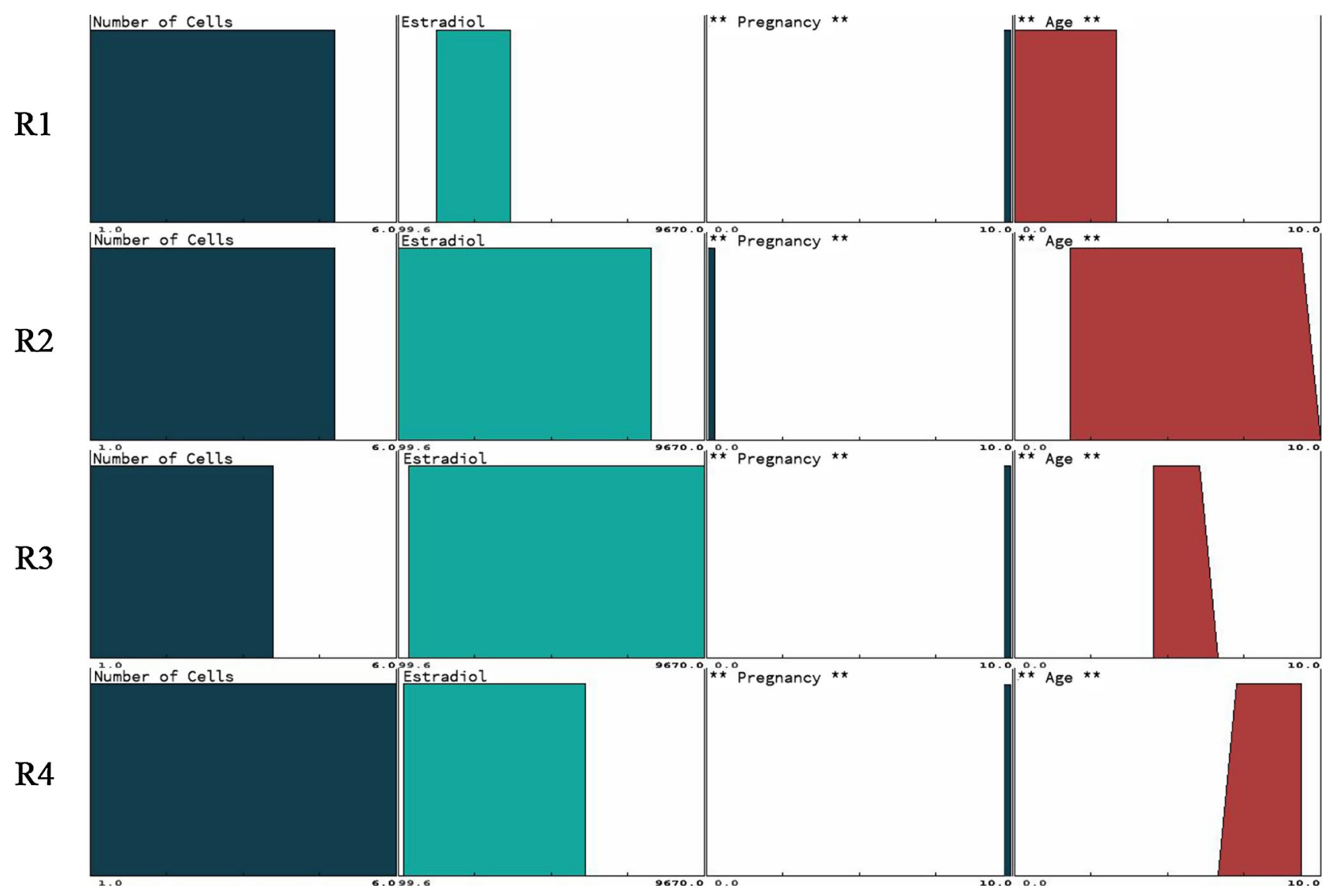

Fig. 6 Rules from experiment 1 -combination 2

- R2 shows interesting information because of its qualitative behavior pattern in women with high estradiol value. It corresponds to women with a medium number of cells, aged very localized around the medium value (approx. 35 years old) with any pregnancy outcome.

- The other rules show others qualitative behaviors of number of cells, patient age and pregnancy results related to the remaining estradiol values.

- EXP2_COMB1 (Antecedent: MA-MES-MH, Consequence: Number of Oocytes) (Fig. 9).

\section{- Notes}

- The antecedent parameters are discrete. These values have been codified as correlative numbers from 1 to the number of different medicaments with the aim to process them like continuous numbers and to realize diffused projections that allow qualitative patterns.

- The value 0 in antecedent parameters mean that it has not been applied any medication of this type.
- The fuzzy sets obtained in the antecedent must be analyzed taking into account that they are discrete attributes and therefore continuous values in this case do not exist. For example, values between 1 and 2, since both values are actually two specific medicaments.

- With the aim of understanding with a high level of details the obtained results, Table 1 shows the various medicaments used in each of the parameters of the antecedent (MA: Parameter 1, MES: Parameter 2, MH: Parameter 3)

\section{- Rules}

- R1 shows that patients with the highest oocytes values are those who have not received the first supplied medication (MA), those who have received the stimulation one or five to eleven stimulation medication (MES) and have received the number two HCG medication.

- R2 indicates that values somewhat higher than average of oocytes (between 25 and 30 approx.) is given in patients that have not received any MA medication, MES medications between two and twelve and any of MH medications. In this rule, 


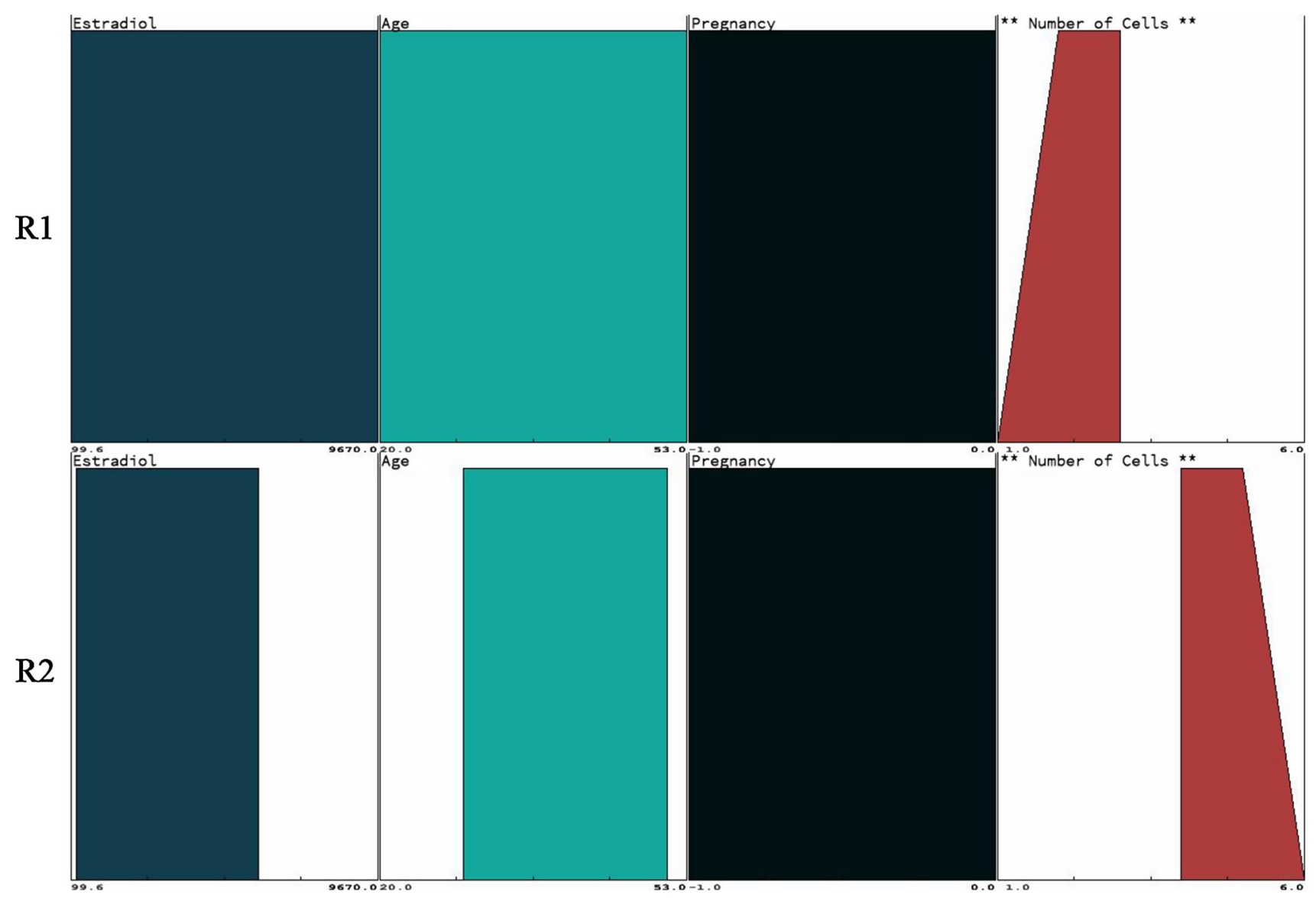

Fig. 7 Rules from experiment 1 -combination 3

although the MES parameters reaches zero, the first value with maximum ownership is equals two.

- R3 shows that values higher than average of oocytes (between 30 and 40 approx.) is given in patients that have not received any MA medication, MES medications between two and twelve and one to three MH medications. In this rule, although the MES parameters reaches one, the first value with maximum ownership is equals two.

- R4 and R6 shows that patient who have not received the MA medication and have received either MES or MH medications have a lower oocytes values than the average (between 11 and 17 approx.).

- R5 and R7 shows that patient who have not received MA, who have received any MES except number twelve and who have received any MH have a medium oocytes value (between 17 and 25).

- $\mathbf{R 8}$ shows that patients with the lowest oocytes value are those who have received MA number zero or number one and either MES or MH medications.

Final section will present the conclusions and future works. In this section and thanks to the experience that the INEBIR fertility clinic offers, the rules are studied in depth for extracting the knowledge that they provide.

\section{Conclusions and future work}

In this work, the application of the data mining tool PreFurGe to a real-world dataset of patients that suffered a IVF treatment has been presented. PreFurGe is a very powerful tool that it is applicable to many different domains, as explained in Sect. 2. One hundred and twenty-three patients (123) and five hundred and fifty-nine (559) treatments applied were analyzed.

Studying in depth the rules resulted after the application of the algorithm to the dataset, some conclusions are obtained. The pregnancy probability is given in women with a certain number of ova. The pregnancy rate is lower with a low or too high number of ova. Through the experience of INEBIR it 


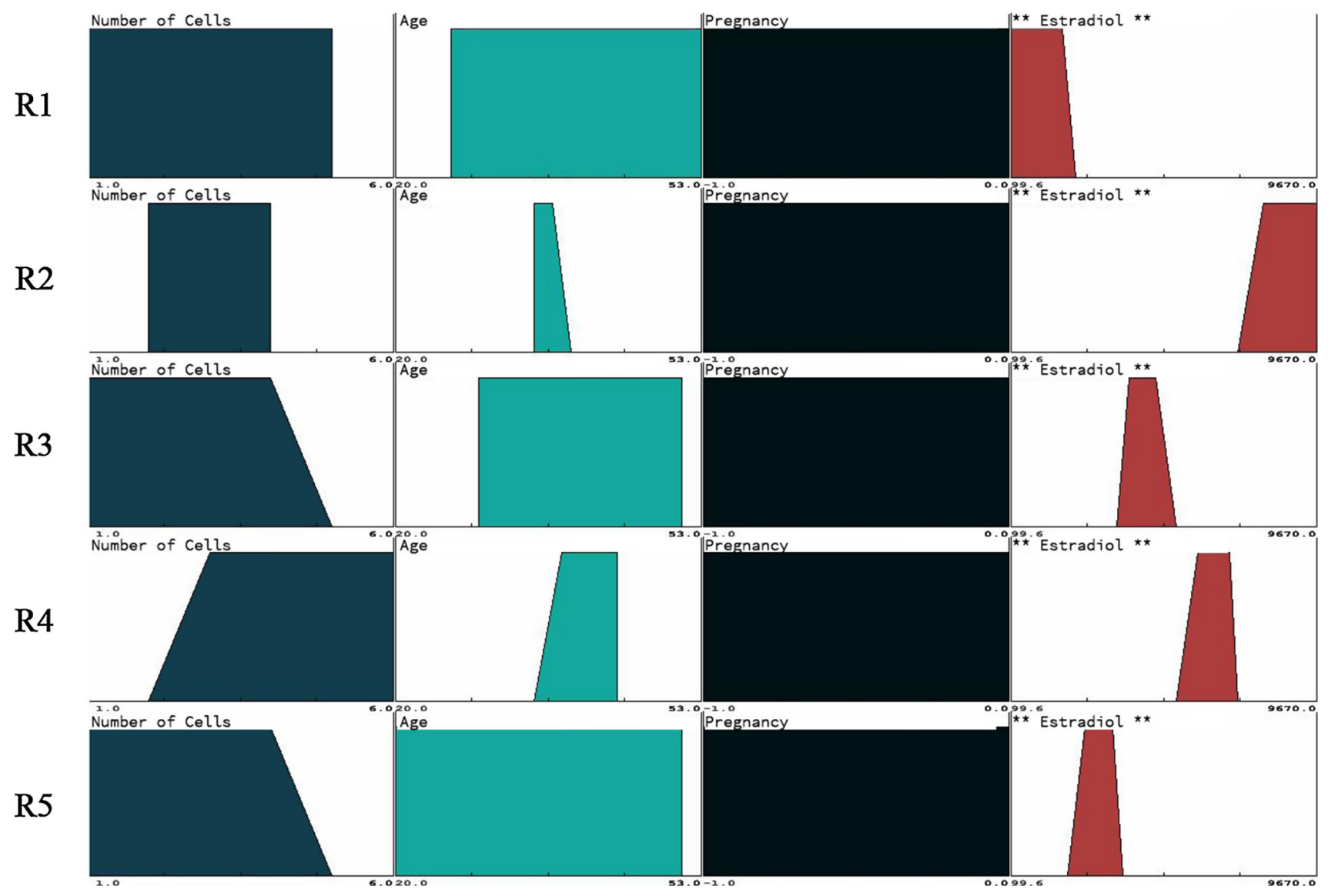

Fig. 8 Rules from experiment 1 -combination 4

has been observed that the higher rate of pregnancy occurs in women with a range of 7-14 ova retrieved. The pregnancy rate decreases when the number of ova is less than 7 or greater than 20.

The estradiol is a potential marker of oocyte quality. The highest rates of pregnancy are given in average levels of estradiol. Low levels of estradiol indicate low quality oocytes, conversely, high levels indicate good quality oocytes, but could be a factor that would prevent pregnancy rate increase. Why a high number of oocytes and estradiol tend to have lower rates of gestation? INEBIRS experience supports that when estradiol exceeds a certain limit (arbitrarily 3000), the endometrium is less capable of implanting an embryo, so it would be more practical cryopreserved embryos and transfer them in a subsequent cycle in which the endometrium has been prepared with more physiological levels. One hypothesis for further work could be if low pregnancy rates, in cycles with high levels of estradiol and oocytes, are recovered when all these cycles cryopreserved and transferred in a later cycle. Another hypothesis is checking if the very high levels of estradiol alter the oocyte and embryo quality.

Age is a critical factor. The number of ova is finite, so as older women are, and pregnancy rates will be worse. With an equal number of ova, older women will have lower pregnancy rates. This effect seems to be relevant from the 27 years old.

At any age there are a number of ova and minimum critical value of estradiol for obtain a positive pregnancy result. Young women need fewer number of ova than older women. Estradiol production seems to be a marker of oocyte quality, but not at all ages. In this moment, it emerges the following question: is it possible that in older women estradiol not a good marker and the critical factor is the number of ovules?

The low number of oocytes predicts a low rate of pregnancy at any age. From a clinical point of view, young women who produce a low number of ova suffer a failure of the ovary and have a low ova capital, resulting in a lower pregnancy rate, although the estradiol could be normal. Experiment 2 shows that ovarian stimulation treatments show no difference between them whenever they get a critical number of oocytes. Therapeutic strategies which require medication before stimulation have worse outcomes. It is a fact contrasted in the clinic where women with low ova capital require pretreatment to stimulation to balance hormonal values before starting the stimulation.

Finally, other future research works proposed are: conducting new experiments trying to generate new rules based 


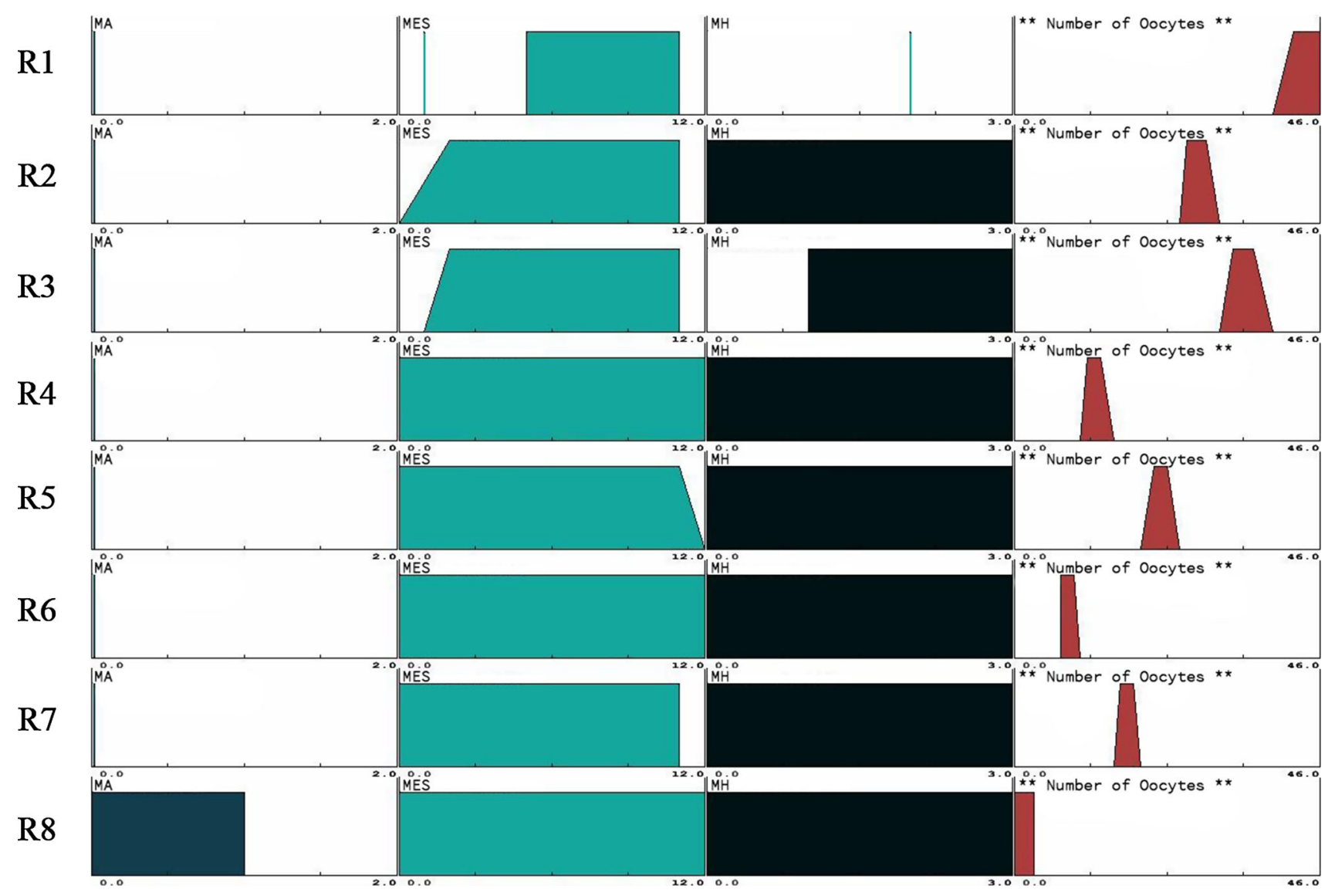

Fig. 9 Rules from experiment 2-combination 1

Table 1 Parameterization values of medicaments

\begin{tabular}{lc}
\hline Medicament & Value \\
\hline Parameter 1 & 1 \\
Decapeptyl mensual 3.75 mg & 2 \\
Procrin $2.8 \mathrm{ml} 14$ dosis & \\
Parameter 2 & 1 \\
Cetrotide $0.25 \mathrm{mg} \times 1$ & 2 \\
Cetrotide $0.25 \mathrm{mg} \times 7$ & 3 \\
Decapeptyl diario $0.1 \mathrm{mg}$ & 4 \\
Gonal-f 1050 & 5 \\
Gonal-f 75 & 6 \\
HMG-Lepori $75 \times 1$ & 7 \\
HMG-Lepori $75 \times 10$ & 8 \\
Menopur $75 \times 10$ amp & 9 \\
Omifin & 10 \\
Orgalutran $0.25 \times 1$ & 11 \\
Procrin $2.8 \mathrm{ml} 14$ dosis & 12 \\
Progynova parches & \\
Parameter 3 & 1 \\
HCG-Lepori 2500,4 amp & \\
Ovitrelle 250 mcg & 2 \\
HCG-Lepori 2500,3 amp & 3
\end{tabular}

on the study of others attributes relevant to the IVF process. It would be interesting to develop an application in which experts could conduct their own experiments independently, with the aim of reducing setup experiment times. Another thing that must be done is the study of alternatives of PreFurGe, contrasting these results and verifying the reliability of the chosen methodology. As far as possible, it is important to continue improving the tool in order to get a better behavior and consequently, better rules generation. Finally, and watching the versatility of PreFurGe, applying it to another different e-Health domain is being considered.

Acknowledgements This research has been supported by the MeGUS project (TIN2013-46928-C3-3-R), Pololas project (TIN2016-76956C3-2-R), by the SoftPLM Network (TIN2015-71938-REDT) of the Spanish the Ministry of Economy and Competitiveness and Fujitsu Laboratories of Europe (FLE). 


\section{References}

Aroba J (2003) Advances in the decision making in software development projects. Ph.D. thesis. University of Seville

Aroba J, Cuadrado-Gallego JJ, Sicilia M-Á, Ramos I, GarcíaBarriocanal E (2008) Segmented software cost estimation models based on fuzzy clustering. J Syst Softw 81:1944-1950

Borges G, Brusamarello V (2015) Sensor fusion methods for reducing false alarms in heart rate monitoring. J Clin Monit Comput 30(6):859-867

Bui DT, Pradhan B, Lofman O, Revhaug I, Dick OB (2012) Spatial prediction of landslide hazards in Hoa Binh province (Vietnam): a comparative assessment of the efficacy of evidential belief functions and fuzzy logic models. Catena 96:28-40

Cotton S, Hill S, Hirstein AF, James JL (2005) Model assisted reproductive technology act. J Gend Race Justice 9:55

Fukuyama Y, Sugeno M (1989) A new method of choosing the number of clusters for fuzzy means method. In: Proceedings of the 5th fuzzy systems symposium, pp 247-250

Furuhashi T, Suzuki T (2001) On interpretability of fuzzy models based on conciseness measure. In: The 10th IEEE international conference on fuzzy systems, 2001, pp 284-287

Gegúndez ME, Aroba J, Bravo JM (2008) Identification of piecewise affine systems by means of fuzzy clustering and competitive learning. Eng Appl Artif Intell 21:1321-1329

Glymour C, Madigan D, Pregibon D, Smyth P (1996) Statistical inference and data mining. Commun ACM 39:35-41

Guillaume S (2001) Designing fuzzy inference systems from data: an interpretability-oriented review. IEEE Trans Fuzzy Syst 9:426-443

Hand DJ (1998) Data mining: statistics and more? Am Stat 52:112-118

Hathaway RJ, Bezdek JC (1993) Switching regression models and fuzzy clustering. IEEE Trans Fuzzy Syst 1:195-204

Hoppner F, Klawonn F (2003) A contribution to convergence theory of fuzzy c-means and derivatives. IEEE Trans Fuzzy Syst 11(5):682694

Josefiok M, Sauer J (2015) Towards an expert system for the field of neurology based on fuzzy logic. In: Joint German/Austrian conference on artificial intelligence (Knstliche Intelligenz), pp 333-340

Jurisica I, Mylopoulos J, Glasgow J, Shapiro H, Casper RF (1998) Casebased reasoning in IVF: prediction and knowledge mining. Artif Intell Med 12:1-24. doi:10.1016/S0933-3657(97)00037-7

Kaufman L, Rousseeuw PJ (2009) Finding groups in data: an introduction to cluster analysis. Wiley, New York

Lawson AK, Klock SC, Pavone ME, Hirshfeld-Cytron J, Smith KN, Kazer RR (2014) Prospective study of depression and anxiety in female fertility preservation and infertility patients. Fertil Steril 102:1377-1384. doi:10.1016/j.fertnstert.2014.07.765
Lopes MHBM, DAncona CAL, Ortega NRS, Silveira PSP, FaleirosMartins AC, Marin HF (2015) A fuzzy logic model for differential diagnosis of lower urinary tract dysfunctions. Int J Urol Nurs 10(3):146-153

Miyahira SA, Araujo E (2008) Fuzzy obesity index for obesity treatment and surgical indication. In: IEEE international conference on fuzzy systems, 2008. FUZZ-IEEE 2008. (IEEE World Congress on Computational Intelligence), pp 2392-2397

Morales DA, Bengoetxea E, Larraaga P, García M, Franco Y, Fresnada M, Merino M (2008) Bayesian classification for the selection of in vitro human embryos using morphological and clinical data. Comput Methods Programs Biomed 90:104-116. doi:10.1016/j. cmpb.2007.11.018

Nakajima H (1996) Fuzzy logic and data mining. In: Proceedings of the 1996 Asian fuzzy systems, IEEE 1996, pp 133-138

Panavaranan P, Wongsawat Y (2013) EEG-based pain estimation via fuzzy logic and polynomial kernel support vector machine. In: Biomedical engineering international conference (BMEiCON), 2013 6th, pp 1-4

Rane AL (2015) Clinical decision support model for prevailing diseases to improve human life survivability. In: 2015 international conference on pervasive computing (ICPC), pp 1-5

SEF (2016) Sociedad Espaola de Fertilidad. Accessed Oct 2016

Shamim MS, Enam SA, Qidwai U (2009) Fuzzy logic in neurosurgery: predicting poor outcomes after lumbar disk surgery in 501 consecutive patients. Surg Neurol 72:565-572

Sugeno M, Yasukawa T (1993) A fuzzy-logic-based approach to qualitative modeling. IEEE Trans Fuzzy Syst 1:7-31. doi:10.1109/ TFUZZ.1993.390281

Tartarisco G, Baldus G, Corda D, Raso R, Arnao A, Ferro M, Gaggioli A, Pioggia G (2012) Personal health system architecture for stress monitoring and support to clinical decisions. Comput Commun 35:1296-1305

Yilmaz A, Ayan K (2011) Risk analysis in breast cancer disease by using fuzzy logic and effects of stress level on cancer risk. Sci Res Essays 6:5179-5191

Yilmaz A, Ayan K (2013) Cancer risk analysis by fuzzy logic approach and performance status of the model. Turk J Electr Eng Comput Sci 21:897-912

Zadeh LA (1965) Fuzzy sets. Inf Control 8:338-353 